\title{
New Knowledge About CCR5, HIV Infection, and Disease Progression: Is "Old" Still Valuable?
}

\author{
Rajeev K. Mehlotra
}

\begin{abstract}
$\mathrm{C}-\mathrm{C}$ chemokine receptor (CCR) 5 (CCR5) is the main HIV-1 coreceptor involved in virus entry and cell-to-cell spread during acute and chronic infections: such CCR5 and T cell tropic viruses are adapted to and replicate in $\mathrm{CD}^{+}$memory $\mathrm{T}$ cells. Polymorphisms in CCR5 regulate CCR5 expression, which, in turn, influences HIV infection acquisition and subsequent disease progression. Among these polymorphisms, a 32-bp deletion in the CCR5 open reading frame (CCR5 $\triangle 32)$ and a single nucleotide polymorphism (SNP) in the promoter $(-2459 \mathrm{G} / \mathrm{A})$ are the most well-characterized polymorphisms. CCR5 $\Delta 32$ provides partial to full protection against HIV infection and, therefore, serves as a basis for gene deletion studies attempting to achieve a permanent HIV cure. Recent studies have discovered that certain SNPs in the CCR region, not within CCR5, also affect CCR5 expression, HIV infection, and disease progression. Although these studies provide further valuable information regarding the role of human genetic variation in HIV/AIDS, they did not incorporate $-2459 \mathrm{G} / \mathrm{A}$. In this article, the author summarizes the knowledge gained through the discovery of these new SNPs and introduces the idea that by not incorporating $-2459 \mathrm{G} / \mathrm{A}$, less comprehensive conclusions may have been reached. Until a strategy that delivers a cure to the millions is found, every piece of information that may help curtail the HIV/AIDS threat to public health should be considered useful.
\end{abstract}

Keywords: CCR5, HIV, host genetics, promoter polymorphism

\section{Human Genetic Variation and HIV/AIDS}

$\mathbf{E}$ ARLY ON IN the HIV epidemic, significant differences in the rate of progression to AIDS were noticed among longitudinally followed HIV-infected persons. The role of the human leukocyte antigen (HLA) system genes in determining the course of disease was established by measuring the $\mathrm{CD}^{+} \mathrm{T}$ cell counts and/or the length of time between HIV infection and development of AIDS. ${ }^{1,2}$ Several HLA genes or haplotypes appear to influence disease progression, although the effects are complex and may depend on interactions with other host and viral genes. ${ }^{3}$ Before the discovery of the role of $\mathrm{C}-\mathrm{C}$ chemokine receptor (CCR) gene polymorphism in HIV infection and disease progression, only genes of the HLA system were thought to influence HIV disease progression. In the 1990s, studies confirmed the protective role of homozygosity for a 32-bp deletion in CCR5 open reading frame (ORF, CCR5 $\Delta 32$ ) against HIV infection. ${ }^{1-3}$ The CCR5 $\Delta 32$ allele is predominantly found in European populations, with no or rare occurrences in Asians and native populations from Africa, the Americas, and Oceania. ${ }^{4}$ The presence of one copy of the deleted CCR5 gene also influences the course of disease, as the onset of AIDS occurs later for some heterozygous persons than for those homozygous for the wild-type (wt) CCR5 gene. ${ }^{1-3}$ The discovery of the role of $C C R 5$ alleles has prompted studies of the possible role of many other host genes in HIV infection and disease progression. ${ }^{5-7}$

\section{A Recent Genome-Wide Association Analysis Uncovered rs 1015164}

A recent study by McLaren $e t$ al. ${ }^{8}$ tested for association between $\sim 8$ million common variants and set-point viral load (spVL) in 6,315 individuals of European ancestry. In this analysis, they found that the top chromosome three single nucleotide polymorphism (SNP) was rs1015164G/A $\left(p=1.5 \times 10^{-19}\right)$. The SNP rs1015164 lies near an antisense transcribed sequence RP11-24F11.2 that overlaps CCR5 and is only weakly correlated with CCR5 $\Delta 32\left(D^{\prime}=0.89, r^{2}=0.03\right)$. Fine mapping of the $1.5 \mathrm{Mb} C C R$ region association signals in the subset of 5,559 individuals, for whom the CCR5 $\triangle 32$ genotype data were available and CCR5 haplotype P1 (Hap-P1,

Center for Global Health and Diseases, Case Western Reserve University School of Medicine, Cleveland, Ohio, USA. 
described hereunder) carriage could be determined, showed that another SNP rs4317138T/C was the top SNP associated with $\operatorname{spVL}\left(p=7.7 \times 10^{-22}\right)$. Interestingly, this SNP highly correlated with the top SNP identified in the analysis of the full sample, rs 1015164G/A $\left(D^{\prime}=1, r^{2}=0.97\right)$. Using conditional association analysis, rs 1015164G/A remained associated with spVL when conditioning on both CCR5 $\triangle 32$ and Hap-P1 (conditional $p=5.2 \times 10^{-4}$ ). McLaren et al. ${ }^{8}$ stated, "These SNPs are located within/near an antisense transcribed sequence that overlaps $C C R 5$ and thus may play a role in regulating its expression. Demonstration of causality of these variants and/or a silencing effect of the antisense transcribed sequence will require functional studies."

\section{New Knowledge About rs1015164, CCR5 Expression, and HIV Outcome}

A more recent study by Kulkarni et al. ${ }^{9}$ has further substantiated the fact that human genetic variation affects HIV infection and disease progression. It also has shown that the role of human genetic variation in HIV/AIDS is not straightforward. The major findings of this study can be summarized as follows: rs1015164 G/A ${ }^{8}$ is in genomic proximity to $R P 11-24 F 11.2$, an antisense long noncoding RNA (lncRNA) gene that overlaps CCR5 and marks expression of the transcript encoded by the gene. Kulkarni et al. ${ }^{9}$ termed this transcript CCR5AS. Their data reveal that higher expression levels of CCR5AS, as a consequence of a variant in an activating transcription factor 1 (ATF1) transcription factor binding site that rs1015164A marks, enhance CCR5 messenger RNA (mRNA) stability, thereby increasing CCR5 mRNA and cell surface expression. The rs1015164 SNP associates with HIV outcome (viral loads and CD4 ${ }^{+}$ $\mathrm{T}$ cell counts) after infection. Thus, the complex interplay among rs1015164A, CCR5AS, and CCR5 provides the functional basis for the association between rs 1015164A and lack of HIV control. Furthermore, these data represent a rare determination of the functional importance of a genome-wide disease association where expression of an lncRNA affects HIV infection and disease progression.

\section{A CCR5 Promoter Polymorphism with Known Functional Significance}

From this complex interplay, an important "old" player missing is CCR5 promoter polymorphism $-2459 \mathrm{G} / \mathrm{A}$ (rs1799987), also known as 59029G/A and 303G/A. In a variety of studies conducted in the 1990s, the $-2459 \mathrm{~A}$ allele, compared with the $-2459 \mathrm{G}$ allele, has been shown to be associated with accelerated HIV disease progression. ${ }^{10,11}$ That the -2459 A allele was associated with significantly higher in vitro promoter activity, CCR5 expression, and HIV propagation, compared with the $-2459 \mathrm{G}$ allele, was then shown in a number of studies. ${ }^{12-14}$ Recent studies have provided molecular mechanisms regarding the association between $C C R 5$ promoter polymorphisms and transcriptional regulation of the promoter, and how this association correlates with CCR5 cell surface expression as well as HIV disease phenotype. ${ }^{15-17}$

The CCR5 haplotype nomenclature system consists of a total of nine polymorphisms, which include CCR5 ORF $\mathrm{wt} / \triangle 32$ and $-2459 \mathrm{G} / \mathrm{A}$. $C C R 5$ haplotypes are organized into nine evolutionarily distinct human haplogroups $(\mathrm{HH})$ designated HHA, $-\mathrm{B},-\mathrm{C},-\mathrm{D},-\mathrm{E},-\mathrm{F}^{*} 1,-\mathrm{F}^{*} 2,-\mathrm{G}^{*} 1$, and $-\mathrm{G}^{*} 2{ }^{18,19}$

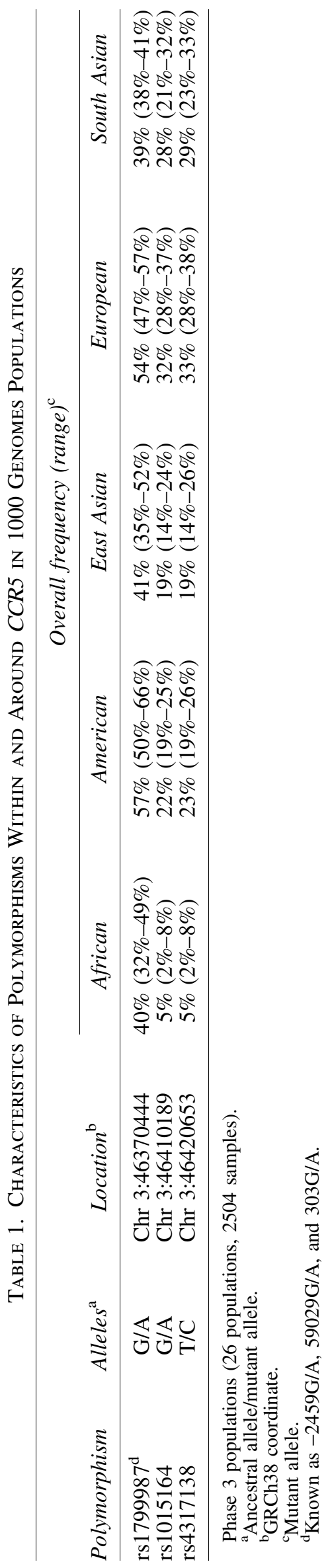


Haplotypes HHA to HHD carry the -2459 G allele, whereas haplotypes HHE to $\mathrm{HHG}^{*} 2$ carry the $-2459 \mathrm{~A}$ allele. ${ }^{18,19}$ Only $\mathrm{HHG}^{*} 2$ carries the $\Delta 32$ allele. HHE, HHF* 1 , and $\mathrm{HHG}^{*} 1$ are grouped together as Hap- $\mathrm{P}^{10}{ }^{10}$ (see Ref. ${ }^{18}$ for further discussion). In the same way that the ORF wt/ $\Delta 32$ and -2459G/A alleles show differences in phenotypic effects in vitro as well as in HIV/AIDS cohorts, different CCR5 haplotypes influence HIV infection and disease outcomes differently. ${ }^{18,20}$ Among all nine CCR5 haplotypes (HHA$\mathrm{HHG}^{*} 2$ ), the consistency of the association of -2459 A allelecarrying HHE homozygosity (E/E genotype) with an unfavorable outcome across diverse populations is noteworthy, which suggests that the HHE haplotype confers similar phenotypic effects against distinct genetic backgrounds. ${ }^{20-24}$

Given this already known functional significance of $-2459 \mathrm{G} / \mathrm{A}$ (hereafter referred to as rs1799987G/A), a likely reason for not including this promoter polymorphism in the key analyses by Kulkarni et al. ${ }^{9}$ could be that the rs 1015164 SNP was found to have a genome-wide effect independent of Hap-P1 (carrying rs1799987A) by McLaren et al. ${ }^{8}$ The analyses by Kulkarni et al. have found that (a) rs1015164A/G variation associates with HIV-1 viral load and $\mathrm{CD} 4^{+} \mathrm{T}$ cell counts across distinct populations (fig. 1 and Supplementary fig. 1) ${ }^{9}$; (b) rs1015164 genotypes (AA/AG and GG) show a significant correlation with CCR5 cell surface expression in bulk memory $\mathrm{CD} 4^{+} \mathrm{T}$ cells and effector memory $\mathrm{CD} 4^{+}$ T cells (fig. 2d) ${ }^{9}$; (c) CCR5AS enhances CCR 5 mRNA and cell surface expression (fig. $3 \mathrm{a}-\mathrm{c})^{9}$; and (d) primary $\mathrm{CD} 4^{+}$ $\mathrm{T}$ cells from rs 1015164AA/AG donors show a considerable increase in infection with $\mathrm{R} 5$ tropic virus as compared with $\mathrm{CD}^{+} \mathrm{T}$ cells from donors with the rs 1015164GG genotype (fig. 7b). ${ }^{9}$ In these analyses, by incorporating rs $1799987 \mathrm{G} / \mathrm{A}$ genotype information, one could have gained an important complementary, more comprehensive insight regarding the outcome. For example, what happens to CCR5 expression, HIV-1 viral loads, and $\mathrm{CD}^{+}{ }^{+} \mathrm{T}$ cell counts in individuals who carry both rs1015164A and rs1799987A alleles? Such individuals may have much higher CCR5 expression, higher viral loads, and decreased $\mathrm{CD} 4^{+} \mathrm{T}$ cell counts than those who carry either the rs1015164A or the rs 1799987A allele. If that is the case, will they be considered as genetically "higher risk" individuals? Therefore, an examination of how rs1799987G/A polymorphism may have influenced those conclusions seems warranted.

\section{A Population Genetics View}

\section{Allele frequencies and linkage disequilibrium}

From a population genetics angle, one may ask, how probable it is that both rs1015164A and rs 1799987A alleles occur together by chance, to perform meaningful analyses? Using the 1000 Genomes populations, the allele frequencies (Table 1) and linkage disequilibrium (LD; Table 2) values suggest that it is reasonably probable in certain populations. Note that the rs 1799987A allele is highly prevalent all across the world (frequencies 32\%-66\%), whereas the rs1015164A allele has frequencies of $14 \%-37 \%$ in most populations and $2 \%-8 \%$ in those from Africa (Table 1). In the Japanese

Table 2. Linkage Disequilibrium Between Polymorphisms Within and Around CCR5 in 1000 Genomes Populations

\begin{tabular}{|c|c|c|c|}
\hline \multirow[b]{2}{*}{ Population } & \multirow[b]{2}{*}{ Description } & \multicolumn{2}{|c|}{ Polymorphism pair } \\
\hline & & $\begin{array}{c}r s 1015164-r s 1799987 \\
\mathrm{D}^{\prime}\left(\mathrm{r}^{2}\right)\end{array}$ & $\begin{array}{c}r s 4317138-r s 1799987 \\
\mathrm{D}^{\prime}\left(\mathrm{r}^{2}\right)\end{array}$ \\
\hline \multirow[t]{5}{*}{ African } & African Caribbean in Barbados (ACB) & $1(0.11)$ & $1(0.11)$ \\
\hline & African Ancestry in southwest United States (ASW) & $1(0.08)$ & $1(0.08)$ \\
\hline & Gambian in Western Division, The Gambia (GWD) & $0.86(0.10)$ & $0.86(0.10)$ \\
\hline & Mende in Sierra Leone (MSL) & $1(0.05)$ & - \\
\hline & Yoruba in Ibadan, Nigeria (YRI) & $0.75(0.05)$ & - \\
\hline \multirow[t]{4}{*}{ American } & Colombian in Medellin, Colombia (CLM) & $1(0.26)$ & $0.82(0.21)$ \\
\hline & Mexican ancestry in Los Angeles, CA (MXL) & $1(0.26)$ & $0.90(0.21)$ \\
\hline & Peruvian in Lima, Peru (PEL) & $0.85(0.09)$ & $0.85(0.09)$ \\
\hline & Puerto Rican in Puerto Rico (PUR) & $1(0.27)$ & $0.94(0.25)$ \\
\hline \multirow[t]{5}{*}{ East Asian } & Chinese Dai in Xishuangbanna, China (CDX) & $1(0.33)$ & $1(0.33)$ \\
\hline & Han Chinese in Beijing, China (CHB) & $1(0.24)$ & $1(0.23)$ \\
\hline & Southern Han Chinese, China (CHS) & $1(0.39)$ & $1(0.39)$ \\
\hline & Japanese in Tokyo, Japan (JPT) & $0.94(0.26)$ & $0.90(0.26)$ \\
\hline & Kinh in Ho Chi Minh City, Vietnam (KHV) & $0.94(0.35)$ & $0.94(0.35)$ \\
\hline \multirow[t]{5}{*}{ European } & $\begin{array}{l}\text { Utah residents with northern and western } \\
\text { European ancestry (CEU) }\end{array}$ & $1(0.42)$ & $0.77(0.29)$ \\
\hline & Finnish in Finland (FIN) & $0.95(0.33)$ & $0.95(0.33)$ \\
\hline & British in England and Scotland (GBR) & $1(0.31)$ & $1(0.31)$ \\
\hline & Iberian population in Spain (IBS) & $1(0.48)$ & $0.90(0.41)$ \\
\hline & Toscani in Italy (TSI) & $0.96(0.39)$ & $0.84(0.31)$ \\
\hline \multirow[t]{5}{*}{ South Asian } & Bengali in Bangladesh (BEB) & $1(0.44)$ & $0.90(0.38)$ \\
\hline & Gujarati Indian in Houston, TX (GIH) & $0.94(0.57)$ & $0.89(0.55)$ \\
\hline & Indian Telugu in the United Kingdom (ITU) & $0.97(0.64)$ & $0.97(0.64)$ \\
\hline & Punjabi in Lahore, Pakistan (PJL) & $0.87(0.49)$ & $0.84(0.49)$ \\
\hline & Sri Lankan Tamil in the United Kingdom (STU) & $1(0.60)$ & $0.97(0.58)$ \\
\hline
\end{tabular}

—Data not available. 
(JPT, $n=104 ;$ rs1015164A, 24\%; rs1799987A, 52\%) and European American (CEU, $n=99$; rs1015164A, 32\%; rs $1799987 \mathrm{~A}, 53 \%$ ) populations, the two alleles are expected to occur together in $12 \%$ and $17 \%$ of the individuals, respectively. These populations were well represented in the study by Kulkarni et al. ${ }^{9}$ (JPT, $n=504$ ) and in the previous study by McLaren et al. ${ }^{8}$ (Europeans, $n=5,559$ ), where the rs $1015164 \mathrm{~A}$ allele frequencies were $27 \%$ and $31 \%$, respectively, highly similar to those reported for the 1000 Genomes populations. Assuming that the rs1799987A allele frequencies in those populations ${ }^{8,9}$ are the same as in the 1000 Genomes populations (JPT, 52\%; CEU, 53\%), the two alleles are expected to occur together in $14 \%(n=71)$ and $16 \%$ $(n=889)$ of the individuals, respectively.

In the study by Kulkarni et al., ${ }^{9}$ although the rs1015164A allele was less frequent in the African American patients $(n=992)$, and AA homozygous individuals were rare, patients carrying at least one rs1015164A allele (AA/AG) also had considerably higher viral load and decreased $\mathrm{CD} 4^{+} \mathrm{T}$ cell counts, pointing to a uniform deleterious effect of rs1015164A in HIV-1 infection across distinct populations. Considering the rs 1015164A (2\%-8\%) and rs1799987A (32\%-49\%) allele frequencies in the 1000 Genomes African populations, these two alleles are expected to occur together only in $1 \%-4 \%$ of the individuals, requiring a very large sample size to study their combined effects.

\section{Haplotype frequencies}

The LD between rs1015164 and rs1799987 SNPs suggests that they are not independent of one another in all populations (Table 2). To further substantiate that the rs1015164A and rs1799987A alleles occur together, frequencies of the haplotype containing both variant alleles were calculated using sample genotype data from the 1000 Genomes populations. ${ }^{25}$ Similar to the expected allele frequencies, the haplotype rs1015164A_rs1799987A is estimated to occur in the JPT, CEU, and African populations at frequencies $24 \%, 32 \%$, and $5 \%$, respectively.

\section{Conclusions}

With the new knowledge about rs1015164G/A, including its high correlation with rs4317138T/C, ${ }^{8,26}$ an important question is whether the regulation of CCR5 expression is based on parallel or divergent mechanisms. Where does rs $1799987 \mathrm{G} / \mathrm{A}$ fit into this picture? Note that the LD patterns of rs1015164-rs1799987 and rs4317138-rs1799987 SNP pairs are highly similar (Table 2). Given that these variants are linked to some degree in many populations, could it be that the original reports of rs1799987G/A were tagging the stronger signal at rs 1015164G/A? To answer these questions, studies, such as that by Kulkarni et al., ${ }^{9}$ need to incorporate rs1799987G/A (-2459G/A) information, due to a mechanistic or constitutive explanation that this SNP already provides. The incorporation of this polymorphism is also important to test the hypothesis that individuals who carry both rs1015164A and rs1799987A alleles (the A_A haplotype) have much higher CCR5 expression, higher viral loads, and decreased $\mathrm{CD} 4^{+} \mathrm{T}$ cell counts than those who carry either the rs1015164A or the rs1799987A allele. Since there are large human genomic association data sets available, it seems possible to test this hypothesis; this analysis is beyond the scope of this article. Finally, considering the status of these polymorphisms may be important also in studies wherein new immunologic $^{27,28}$ and chemotherapeutic ${ }^{29,30}$ strategies are evaluated. In those studies, knowing whether an individual, receiving such an immunologic or chemotherapeutic intervention, is carrying two, one, or no A_A haplotype would enable a better understanding of the response to the intervention. In this era of seeking an HIV cure, where all the emphasis has been on the 32-bp deletion, it seems that the well-described promoter polymorphism, -2459G/A, has been forgotten or ignored. ${ }^{20}$ Until we find a strategy that delivers a cure to the millions, we should make use of every piece of information that may help curtail the HIV/AIDS threat to public health.

\section{Acknowledgments}

This article is the result of inspiration and guidance from the Higher Self, and is dedicated to the loving memories of the late Dr. Anil Ghosh, a fellow researcher and a dear friend. The author is grateful to Jasmine Olvany, Dr. Ricky Chan, Dr. Carolyn Myers, Marlin Linger, and Quentin Watson for commenting on the article.

\section{Author Contributions}

R.K.M. wrote the article.

\section{Author Disclosure Statement}

No competing financial interests exist.

\section{Funding Information}

No funding was received for this article.

\section{References}

1. McLaren PJ, Carrington M: The impact of host genetic variation on infection with HIV-1. Nat Immunol 2015;16: 577-583.

2. Naranbhai V, Carrington M: Host genetic variation and HIV disease: From mapping to mechanism. Immunogenetics 2017;69:489-498.

3. Tough RH, McLaren PJ: Interaction of the host and viral genome and their influence on HIV disease. Front Genet 2019;9:720.

4. Solloch UV, Lang K, Lange V, Böhme I, Schmidt AH, Sauter J: Frequencies of gene variant CCR5- $\Delta 32$ in 87 countries based on next-generation sequencing of 1.3 million individuals sampled from 3 national DKMS donor centers. Hum Immunol 2017;78:710-717.

5. Stephens HA: Immunogenetic surveillance of HIV/AIDS. Infect Genet Evol 2012;12:1481-1491.

6. Martin MP, Carrington M: Immunogenetics of HIV disease. Immunol Rev 2013;254:245-264.

7. Mehlotra RK, Zimmerman PA, Weinberg A: Defensin gene variation and HIV/AIDS: A comprehensive perspective needed. J Leukoc Biol 2016;99:687-692.

8. McLaren PJ, Coulonges C, Bartha I, et al.: Polymorphisms of large effect explain the majority of the host genetic contribution to variation of HIV-1 virus load. Proc Natl Acad Sci USA 2015;112:14658-14663. 
9. Kulkarni S, Lied A, Kulkarni V, et al: CCR5AS lncRNA variation differentially regulates CCR5, influencing HIV disease outcome. Nat Immunol 2019;20:824-834.

10. Martin MP, Dean M, Smith MW, et al.: Genetic acceleration of AIDS progression by a promoter variant of CCR5. Science 1998;282:1907-1911.

11. McDermott DH, Zimmerman PA, Guignard F, Kleeberger CA, Leitman SF, Murphy PM: CCR5 promoter polymorphism and HIV-1 disease progression. Multicenter AIDS Cohort Study (MACS). Lancet 1998;352:866-870.

12. Shieh B, Liau YE, Hsieh PS, Yan YP, Wang ST, Li C: Influence of nucleotide polymorphisms in the $C C R 2$ gene and the CCR5 promoter on the expression of cell surface CCR5 and CXCR4. Int Immunol 2000;12:1311-1318.

13. Salkowitz JR, Bruse SE, Meyerson H, et al.: CCR5 promoter polymorphism determines macrophage CCR5 density and magnitude of HIV-1 propagation in vitro. Clin Immunol 2003;108:234-240.

14. Kawamura T, Gulden FO, Sugaya M, et al.: R5 HIV productively infects Langerhans cells, and infection levels are regulated by compound CCR5 polymorphisms. Proc Natl Acad Sci USA 2003;100:8401-8406.

15. Jiang D, Mummidi S, Ahuja SK, Jarrett HW: CCR5 promoter haplotype transcription complex characterization. J Health Care Poor Underserved 2011;22:73-90.

16. Gornalusse GG, Mummidi S, Gaitan AA, et al.: Epigenetic mechanisms, T-cell activation, and CCR5 genetics interact to regulate T-cell expression of CCR5, the major HIV-1 coreceptor. Proc Natl Acad Sci USA 2015;112:E4762-E4771.

17. Joshi A, Punke EB, Sedano M, et al: CCR5 promoter activity correlates with HIV disease progression by regulating CCR5 cell surface expression and CD4 T cell apoptosis. Sci Rep 2017;7:232.

18. Gonzalez E, Bamshad M, Sato N, et al.: Race-specific HIV1 disease-modifying effects associated with CCR5 haplotypes. Proc Natl Acad Sci USA 1999;96:12004-12009.

19. Mummidi S, Bamshad M, Ahuja SS, et al.: Evolution of human and non-human primate $\mathrm{CC}$ chemokine receptor 5 gene and mRNA. Potential roles for haplotype and mRNA diversity, differential haplotype-specific transcriptional activity, and altered transcription factor binding to polymorphic nucleotides in the pathogenesis of HIV-1 and simian immunodeficiency virus. J Biol Chem 2000;275:18946-18961.

20. Mehlotra RK: CCR5 promoter polymorphism $-2459 \mathrm{G}>\mathrm{A}$ : Forgotten or ignored? Cells 2019;8:651.

21. Mehlotra RK, Hall NB, Bruse SE, et al.: CCR2, CCR5, and CXCL12 variation and HIV/AIDS in Papua New Guinea. Infect Genet Evol 2015;36:165-173.
22. Malhotra R, Hu L, Song W, et al:: Association of chemokine receptor gene (CCR2-CCR5) haplotypes with acquisition and control of HIV-1 infection in Zambians. Retrovirology 2011;8:22.

23. Coloccini RS, Dilernia D, Ghiglione Y, et al.: Host genetic factors associated with symptomatic primary HIV infection and disease progression among Argentinean seroconverters. PLoS One 2014;9:e113146.

24. Jaumdally SZ, Picton A, Tiemessen CT, et al.: CCR5 expression, haplotype and immune activation in protection from infection in HIV-exposed uninfected individuals in HIV-serodiscordant relationships. Immunology 2017;151: 464-473.

25. Li Z, Zhang Z, He Z, et al.: A partition-ligationcombination-subdivision EM algorithm for haplotype inference with multiallelic markers: Update of the SHEsis (http://analysis.bio-X.cn). Cell Res 2009;19:519-523.

26. Ensembl Genome Browser. http://useast.ensembl.org/Homo_ sapiens/Variation/HighLD?db=core;r=3:46420153-46421153; $\mathrm{v}=\mathrm{rs} 4317138 ; \mathrm{vdb}=$ variation; $\mathrm{vf}=1567156$; second_variant_ name $=$ rs1015164 accessed May 25, 2020.

27. Mendoza P, Gruell H, Nogueira L, et al.: Combination therapy with anti-HIV-1 antibodies maintains viral suppression. Nature 2018;561:479-484.

28. Bar-On Y, Gruell H, Schoofs T, et al.: Safety and antiviral activity of combination HIV-1 broadly neutralizing antibodies in viremic individuals. Nat Med 2018;24:17011707.

29. Manak MM, Moshkoff DA, Nguyen LT, et al.: Anti-HIV-1 activity of the neurokinin-1 receptor antagonist aprepitant and synergistic interactions with other antiretrovirals. AIDS 2010;24:2789-2796.

30. Tebas P, Tuluc F, Barrett JS, et al.: A randomized, placebo controlled, double masked phase IB study evaluating the safety and antiviral activity of aprepitant, a neurokinin-1 receptor antagonist in HIV-1 infected adults. PLoS One 2011;6:e24180.

Address correspondence to: Rajeev K. Mehlotra

Center for Global Health and Diseases

Case Western Reserve University School of Medicine Biomedical Research Building, \#409A

2109 Adelbert Road Cleveland, $\mathrm{OH} 44106$

USA

E-mail: rkm@case.edu 